\title{
BMJ Open Long-term outcomes of urinary tract infection (UTI) in Childhood (LUCI): protocol for an electronic record-linked cohort study
}

Fiona V Lugg-Widger, ${ }^{0}$ Lianna Angel, ${ }^{1}$ Rebecca Cannings-John, ${ }^{1}$ Hywel Jones, ${ }^{2}$ Mandy Lau, ${ }^{1}$ Christopher Butler, ${ }^{3}$ Nick A Francis, ${ }^{4}$ Alastair D Hay, ${ }^{5}$ Margaret Heginbothom, ${ }^{6}$ Kerenza Hood, ${ }^{1}$ Shantini Paranjothy, ${ }^{2}$ Judith Vandervoort, ${ }^{7}$ Kathryn Hughes ${ }^{4}$

To cite: Lugg-Widger FV, Angel L, Cannings-John R, et al. Long-term outcomes of urinary tract infection (UTI) in Childhood (LUCI): protocol for an electronic record-linked cohort study. BMJ Open 2019;9:e024210. doi:10.1136/ bmjopen-2018-024210

- Prepublication history for this paper is available online. To view these files, please visit the journal online (http://dx.doi. org/10.1136/bmjopen-2018024210).

Received 15 May 2018 Revised 14 January 2019 Accepted 8 February 2019

Check for updates

(C) Author(s) (or their employer(s)) 2019. Re-use permitted under CC BY-NC. No commercial re-use. See rights and permissions. Published by BMJ.

For numbered affiliations see end of article.

Correspondence to Dr Fiona V Lugg-Widger; LuggFV@cardiff.ac.uk

\section{ABSTRACT}

Introduction Current guidelines advise the prompt diagnosis and treatment of urinary tract infection (UTI) in children to improve both short and longer term outcomes. However, the risk of long-term complications following childhood UTI is unclear. UTI is relatively common but difficult to diagnose in children as symptoms are nonspecific. Diagnosis requires a urine sample, but sampling is difficult and infrequent, and it is not clear if sampling should be given greater priority in primary care. The LUCI study will assess the short, medium and longer term outcomes of childhood UTI associated with routine and systematic sampling practices.

Methods and analysis Two data sets will be established. The first will consist of routinely collected data (hospital, general practice (GP), microbiology) from children born and resident in Wales, linked via the Secure Anonymised Information Linkage (SAIL) Databank (an 'e-cohort'). Urine sampling in this data set reflects normal practice 'routine sampling'. Outcomes (including renal scarring, hypertension, end-stage renal failure, hospital admissions, GP consultations, antibiotic prescriptions) for children with at least one UTI confirmed with microbiological culture (mcUTI) or no mcUTI before the age of 5 will be compared. The second will combine data from two prospective observational studies ('DUTY' and 'EURICA') employing systematic urine sampling for children presenting to primary care with acute, undifferentiated illness, linked to routine data via SAIL (Wales) and NHS Digital (England). Outcomes (as above, plus features of mcUTI) for children with an mcUTI in this data set, identified through systematic urine sampling, will be compared with those with an mcUTI identified through routine urine sampling (data set 1).

Ethics and dissemination The study protocol has been approved by NHS Wales Research Ethics Committee and the Health Research Authority's Confidentiality Advisory Group. Methods of innovative study design and findings will be disseminated through peer-review journals and conferences. Results will be of interest to clinical and policy stakeholders in the UK.

\section{Strengths and limitations of this study}

- Use of routinely collected data in the study allows the identification of rare chronic outcomes, from large numbers of children at risk.

- This multisourced data set will allow a comparison of outcomes over 5 years for children with and without microbiologically confirmed urinary tract infection according to routine clinical practice; and compare outcomes in these groups with those observed in high-quality research data using systematic urine sampling.

- This study will help prioritise interventions to improve early diagnosis, sampling and treatment, potentially improving health outcomes and reducing National Health Service costs.

- Using routinely collected data relies on the quality of coding and availability of data.

- Using routinely collected data limits the information available on the children and their outcomes.

\section{INTRODUCTION}

Urinary tract infections (UTI) are a common cause of acute illness in children and an important contributor to hospital admissions for serious bacterial infection. ${ }^{1-7}$ In UK primary care, UTI is the cause of approximately $6 \%$ of acute illness consultations in children less than 5 years old. ${ }^{57}$

Childhood UTI can lead to renal scarring. ${ }^{689}$ Renal scarring has been linked with long-term complications including hypertension, pre-eclampsia and renal failure. ${ }^{10-12}$ Clinical guidelines promote the prompt diagnosis and treatment of UTI as a measure to prevent renal scarring and longer term complications. ${ }^{6}$ It is not clear what the risks of longer term complications are for children with UTI. A systematic review in 2010 found that the prevalence of 
renal scarring following first childhood UTI was $15 \% .^{8}$ Most included studies were conducted in secondary care and most required fever for inclusion in the study. ${ }^{8}$ These are likely to represent more serious UTIs than those presenting in primary care, and this rate of renal scarring may not apply to all children presenting with UTI. The risk of long-term complications (such as hypertension and renal failure) as a result of renal scarring following childhood UTI is also not clear, with some researchers questioning this association. ${ }^{61314}$ A review, commissioned by the National Institute for Health and Care Excellence (NICE), concluded that 'there are no appropriate studies that accurately estimate the risks of long term complications as a result of childhood UTI. ${ }^{6}$

A urine sample is necessary to confirm the presence of UTI in childhood as the presenting symptoms are non-specific and similar to those found in many common childhood illnesses (eg, respiratory infections, gastroenteritis, tonsillitis, ear infections) such as high temperature, poor feeding, vomiting, lethargy and irritability. ${ }^{6}$ Furthermore, microbiological confirmation is important as some children with UTI require invasive investigations. ${ }^{6}$ Children who become seriously ill and who are assessed in emergency departments or admitted to hospital will usually have their urine sampled. ${ }^{1}$ However, in primary care, where most acute illness is seen, urine is infrequently sampled. It is estimated that urine is sampled in fewer than $2 \%$ of acute illness consultations with children under 5 years old in the UK. ${ }^{4}$ Studies have suggested that many cases of childhood UTI are missed in primary care. ${ }^{15-17}$ Routine practice (urine sampling based on clinician suspicion) is likely to miss more than two-thirds of UTIs in primary care. ${ }^{57}$ The clinical implications of missed childhood UTIs are not known. Increasing urine sampling in primary care is likely to increase the diagnosis of childhood microbiologically confirmed UTI (mcUTI), and is advocated by current guidelines, but it is not clear whether this is an appropriate strategy, whether outcomes for children would improve or to what extent it should be increased. ${ }^{4-6} 1518$

Clarification is needed on two issues: First, to determine what the longer term outcomes are following childhood UTI (including those identified in primary care), and second, to determine whether outcomes vary according to sampling practice.

In this study we will describe clinical outcomes for all children with one or more mcUTIs aged less than 5 years old, compared with those with no mcUTI, using National Health Service (NHS) laboratory data from across Wales. We will examine the risk factors for being diagnosed with renal scarring following mcUTI.

We will also describe longer term follow-up of clinical outcomes for at least 5 years following participation in two UK prospective cohort studies of acutely ill children with systematic urine sampling in primary care, the Diagnosis of Urinary Tract infection in Young children (DUTY) and EURICA (a Welsh cohort study of urinary tract infection in children) studies. ${ }^{57}$ We will compare the outcomes of those with mcUTI identified through these studies (systematic urine sampling) with those identified through routine practice.

\section{METHODS AND DESIGN \\ Research objectives}

The Long-term outcomes of UTI in Childhood (LUCI) study will use data linkage of routinely collected data sets and data from two cohorts of participants to answer two main research questions:

Research question 1: Through routine sampling, do children who have experienced a mcUTI aged $<5$ years old have worse outcomes (medium ( $0-5$ years); longer term $(>5$ years $)$ ) compared with children who have not experienced an mcUTI?

Research question 2: Are short-term ( $<12$ months) and medium-term (1-4 years) outcomes different for children with childhood mcUTI identified through systematic sampling compared with routine sampling (standard, clinician-led sampling)?

\section{Study design}

This is a data linkage study comprising two overarching data sets of children. Data set 1 will comprise routinely collected health data from children born and resident in Wales. Children in this data set will have had urine sampled according to routine practice. Routine data will be available on all children for 7 years, and longer for some (ie, children will be followed up until the date of data abstraction). Data set 1 will be used to answer research question 1 .

Data set 2 will be children who participated in the EURICA or DUTY studies. Children in this data set had their urine systematically sampled (all children presenting with an acute illness were asked to provide a urine sample). DUTY and EURICA children will be followed up by linking records to routinely collected health data from England (using NHS Digital) and Wales (using Secure Anonymised Information Linkage, SAIL). Follow-up will be for a minimum of 5 years, and longer where data are available. Data set 2 will be used to describe longer term follow-up for DUTY and EURICA study children. Those with mcUTI in data set 2 will be compared with those with mcUTI identified in data set 1 to answer research question 2.

The study formally started in October 2016 and will report to funder in June 2019. A summary of the data sources is provided in table 1 .

\section{Data providers and data sets \\ The EURICA and DUTY studies}

This work builds on two large cohort studies of acutely ill children, aged less than 5 years old, presenting in primary care, in which mcUTI status was determined using systematic urine sampling. ${ }^{57}$ In both studies, clinical and demographic data were collected and urine samples requested from all children included in the 


\begin{tabular}{|c|c|c|c|c|c|}
\hline \multirow[b]{2}{*}{ Data provider } & \multirow[b]{2}{*}{ Data source } & \multirow[b]{2}{*}{ Dates available } & \multirow[b]{2}{*}{ Indicative/key data items } & \multicolumn{2}{|l|}{ Data set } \\
\hline & & & & $\begin{array}{l}\text { Data set } \\
1 \text { (routine } \\
\text { sampling) }\end{array}$ & $\begin{array}{l}\text { Data set } 2 \\
\text { (systematic } \\
\text { sampling) }\end{array}$ \\
\hline \multirow[t]{5}{*}{ SAIL (Wales) } & GP & $\begin{array}{l}\text { January } 1994 \text { to } \\
\text { October } 2016\end{array}$ & $\begin{array}{l}\text { Secondary outcomes } \\
\text { including antibiotic } \\
\text { prescriptions, GP } \\
\text { consultations, chronic } \\
\text { kidney disease, } \\
\text { hypertension }\end{array}$ & $\checkmark$ & $\checkmark$ \\
\hline & $\begin{array}{l}\text { Patient Episode Database for } \\
\text { Wales (PEDW) }\end{array}$ & $\begin{array}{l}\text { January } 1994 \text { to } \\
\text { April } 2017\end{array}$ & $\begin{array}{l}\text { Primary and secondary } \\
\text { outcomes and covariates } \\
\text { including renal scarring, } \\
\text { hospital admission, end- } \\
\text { stage renal failure, VUR, } \\
\text { renal/bladder surgery }\end{array}$ & $\checkmark$ & $\checkmark$ \\
\hline & $\begin{array}{l}\text { Welsh Electronic Cohort of } \\
\text { Children (WECC) }\end{array}$ & $\begin{array}{l}\text { January } 1994 \\
\text { to September } \\
2011\end{array}$ & $\begin{array}{l}\text { Defines a child as 'born in } \\
\text { Wales' }\end{array}$ & $\checkmark$ & $\checkmark$ \\
\hline & $\begin{array}{l}\text { Datastore (data repository } \\
\text { storing microbiology culture } \\
\text { data from the Laboratory } \\
\text { Information Management } \\
\text { Systems in Wales) }\end{array}$ & 2005-2014 & $\begin{array}{l}\text { Defines a microbiologically } \\
\text { confirmed UTI }\end{array}$ & $\checkmark$ & $\checkmark$ \\
\hline & Outpatient data & $\begin{array}{l}\text { January } 1994 \\
\text { to April } 2017\end{array}$ & $\begin{array}{l}\text { Primary outcome-renal } \\
\text { scarring }\end{array}$ & $\checkmark$ & $\checkmark$ \\
\hline $\begin{array}{l}\text { NHS Digital } \\
\text { (England) }\end{array}$ & Outpatient & $\begin{array}{l}\text { April } 2008 \text { to } \\
\text { March } 2017\end{array}$ & $\begin{array}{l}\text { Primary outcome-renal } \\
\text { scarring }\end{array}$ & & $\checkmark$ \\
\hline $\begin{array}{l}\text { Bristol University } \\
\text { (England and } \\
\text { Wales) }\end{array}$ & DUTY & $\begin{array}{l}\text { April } 2010 \text { to April } \\
2012\end{array}$ & $\begin{array}{l}\text { Baseline characteristics, } \\
\text { UTI status, presenting } \\
\text { symptoms and signs, initial } \\
\text { clinical management }\end{array}$ & & $\checkmark$ \\
\hline $\begin{array}{l}\text { Cardiff University } \\
\text { (Wales) }\end{array}$ & EURICA & $\begin{array}{l}\text { July } 2008 \text { to July } \\
2010\end{array}$ & $\begin{array}{l}\text { Baseline characteristics, } \\
\text { UTI status, presenting } \\
\text { symptoms and signs, initial } \\
\text { clinical management }\end{array}$ & & $\checkmark$ \\
\hline
\end{tabular}

DUTY, Diagnosis of Urinary Tract infection in Young children; EURICA, Welsh cohort study of urinary tract infection in children; GP, general practice; SAIL, Secure Anonymised Information Linkage; UTI, urinary tract infection; VUR, vesicoureteric reflux.

study and analysed in NHS microbiology laboratories. In the EURICA study, 1003 children were recruited from general practitioner's (GP) practices in Wales between March 2008 and July 2010. 5.9 per cent of children had mcUTI. Participants had a GP notes review at 6 months. In the DUTY study, 7163 children were recruited from GP practices in England and Wales between April 2010 and April 2012 and 5.6\% children had moUTI confirmed in NHS labs. An algorithm to predict UTI based on presenting symptoms and signs was developed. Neither study had sufficient follow-up to determine whether renal investigations to look for renal scarring had been undertaken or found. EURICA was funded by the National Institute for Social Care 
and Health Research (now Health and Care Research Wales) and sponsored by Cardiff University, and DUTY was funded by the National Institute for Health Research-Health Technology Assessment and sponsored by Bristol University.

\section{SAIL Databank}

The study will use the SAIL Databank to access routinely collected data outlined in table 1 . SAIL is a repository for a broad range of routinely collected health and population data in Wales. SAIL will also act as a data safe haven for the clinical DUTY and EURICA data sets and data made available from NHS Digital and Individual Health Boards. All data will be accessed via the SAIL Gateway following Information Governance Review Panel (IGRP) approval. SAIL Databank does not handle any identifiable data, therefore all data will be anonymised including data transferred from other information centres. ${ }^{19-21}$

\section{NHS Digital}

NHS Digital is the trading name of the Health and Social Care Information Centre. This study will access Hospital Episode Statistics (HES) data for participants of the DUTY and EURICA studies. All available inpatient and outpatient records belonging to each study participant will be requested and approved by the Independent Group Advising on the Release of Data (IGARD) panel. The data requested include diagnosis, procedures and length of episode according to the codes of the 10th Revision of the International Statistical Classification of Diseases and Related Health Problems.

\section{Public Health Wales}

Public Health Wales will provide a data extract of urine microbiology culture results from all microbiology laboratories in Wales (Datastore) for use with this project. This will be transferred to SAIL.

\section{Individual Health Boards}

Health boards in Wales will be approached to access anonymised radiology data for patients in data set 1. A one off data extract of patient-level attendance data for patients born between 1 January 1994 and 31 December 2012 who attended radiology between 1994 and 2016 will be transferred to SAIL. Data extracted include examination performed, attendance data and the radiology report.

\section{Opportunity to opt out (data set 2)}

Data set 1 uses routinely collected data that are fully anonymised so we do not require individual consent in order to access these data. Data set 2 involves participants from the DUTY and EURICA studies and therefore requires section 251 (s251) support of the 2006 NHS Act approval from the Health Research Authority's Confidentiality Advisory Group (CAG) to pass identifiable participant data legally held by Cardiff University and Bristol University (as sponsors of the studies) to the data providers. This is using an opt-out/dissent model instead of obtaining further consent. In order to provide the opportunity for participants to dissent a letter has been developed to be sent to all parents of participants. Participants have the opportunity to contact the study team through an online web form, by email, text or telephone (details provided on website) and register their dissent. These participants who register their dissent will not be included in any of the data sets sent to the information centres and therefore will not appear in the data sets for analysis. A participant representative was consulted on the layout, wording and level of information contained in the participant opt-out letter and on the study website. A key consideration was to communicate the data transfer process. The final letter was approved by both an NHS Research Ethics Committee and CAG committee as part of overall governance approval for the study.

\section{Data matching}

For included data sets, data will be sent to NHS Digital and SAIL (via NHS Wales Informatics Service (NWIS), their trusted third party) for matching using a combination of NHS number, name, address and date of birth. Matching with NHS Digital data will be by exact matching on NHS number, date of birth and postcode. This has been conducted in other studies and achieved a high match rate $(99.6 \%) .{ }^{22}$ NWIS match using NHS number, date of birth, sex and postcode. Data matching by NHS Digital will be separate from matching conducted by NWIS on behalf of SAIL. We would expect only a small number of participants matched to both English and Welsh NHS records; however, there is the possibility of this for those using services across the border to their current address.

\section{The anonymised data set}

For DUTY and EURICA participants, NHS Digital will retain the unique study ID assigned by the DUTY/ EURICA study, with the HES records after the matching process before it is sent to SAIL Databank. This ID will be retained for data transfer to ensure the data can be linked. The same applies to data sent to NWIS. Requested data will then be linked for each participant via SAIL using the unique study ID. The DUTY and EURICA clinical data sets will be transferred to SAIL following a process of deidentification. The data flow is shown in figure 1 . The study ID will be replaced by an anonymised linking field (ALF) to enable all data sets to be linked at an individual level. The resulting data set will contain clinical variables (from DUTY, EURICA, SAIL and NHS Digital), deidentified demographic variables (eg, week of birth and lower super output area) and the ALF. The key between study ID and ALF will be retained and encrypted as a further safeguard. The ALF is further encrypted so that data sets and records cannot be linked across multiple projects a researcher has access to. 


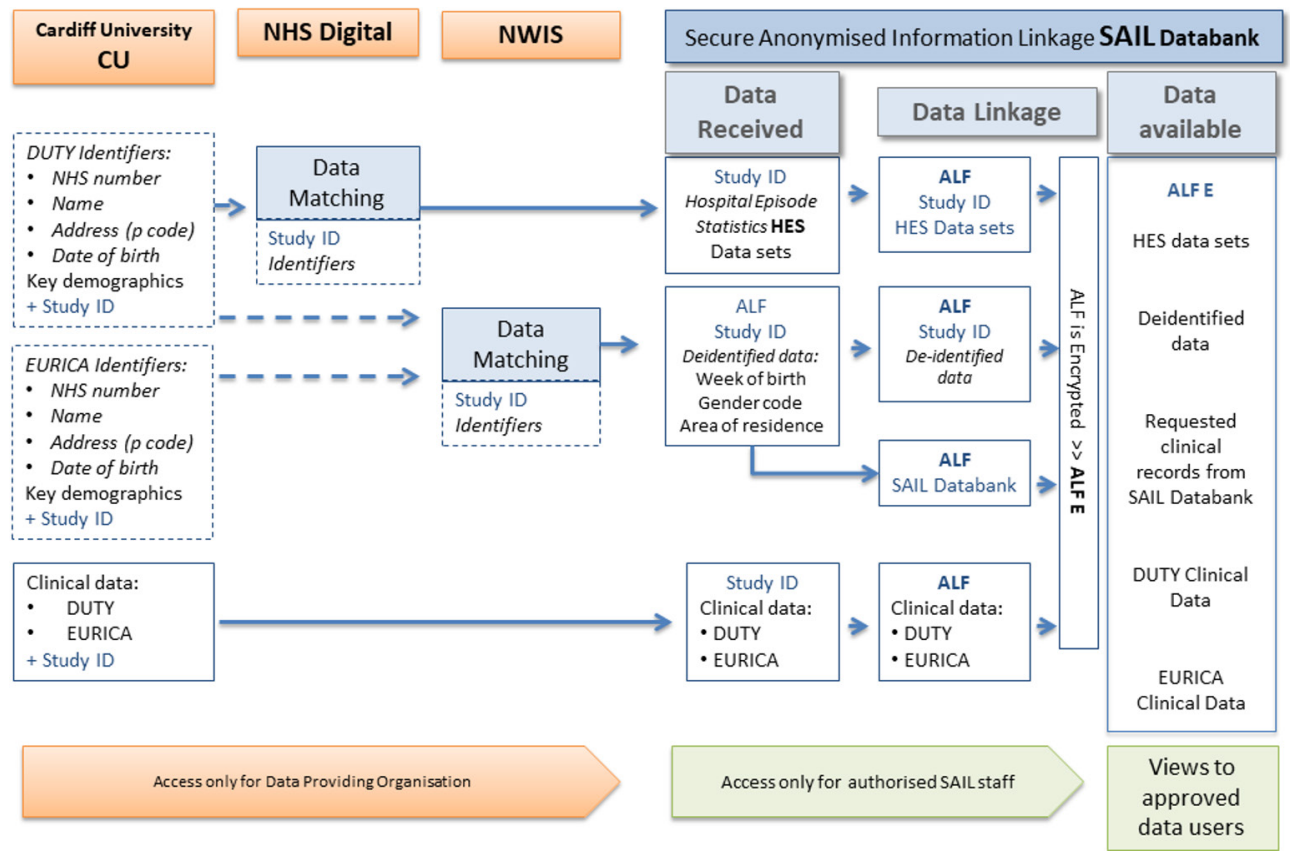

Figure 1 The data flow for data set 2. ALF, anonymised linking field; DUTY, Diagnosis of Urinary Tract infection in Young children; EURICA, Welsh cohort study of Urinary Tract Infection in Children; NHS, National Health Service; NWIS, NHS Wales Informatics Service.

\section{Study participants}

A flow chart of participants in the study is shown in figure 2. Data set 1 (routine sampling) will be children born and resident in Wales for the first 5 years of life; who were less than 5 years old between 1999 and 2012 . The main analysis will be on children born between 1 January 2005 and 31 December 2009 to ensure that all of the first 5 years of life are covered by the dates when Datastore is available.

Data set 2 (systematic sampling from DUTY and EURICA) will be children who participated in the EURICA or DUTY studies, who were not withdrawn from the study and when provided with the opportunity to opt out, remained in the study. For research question 2

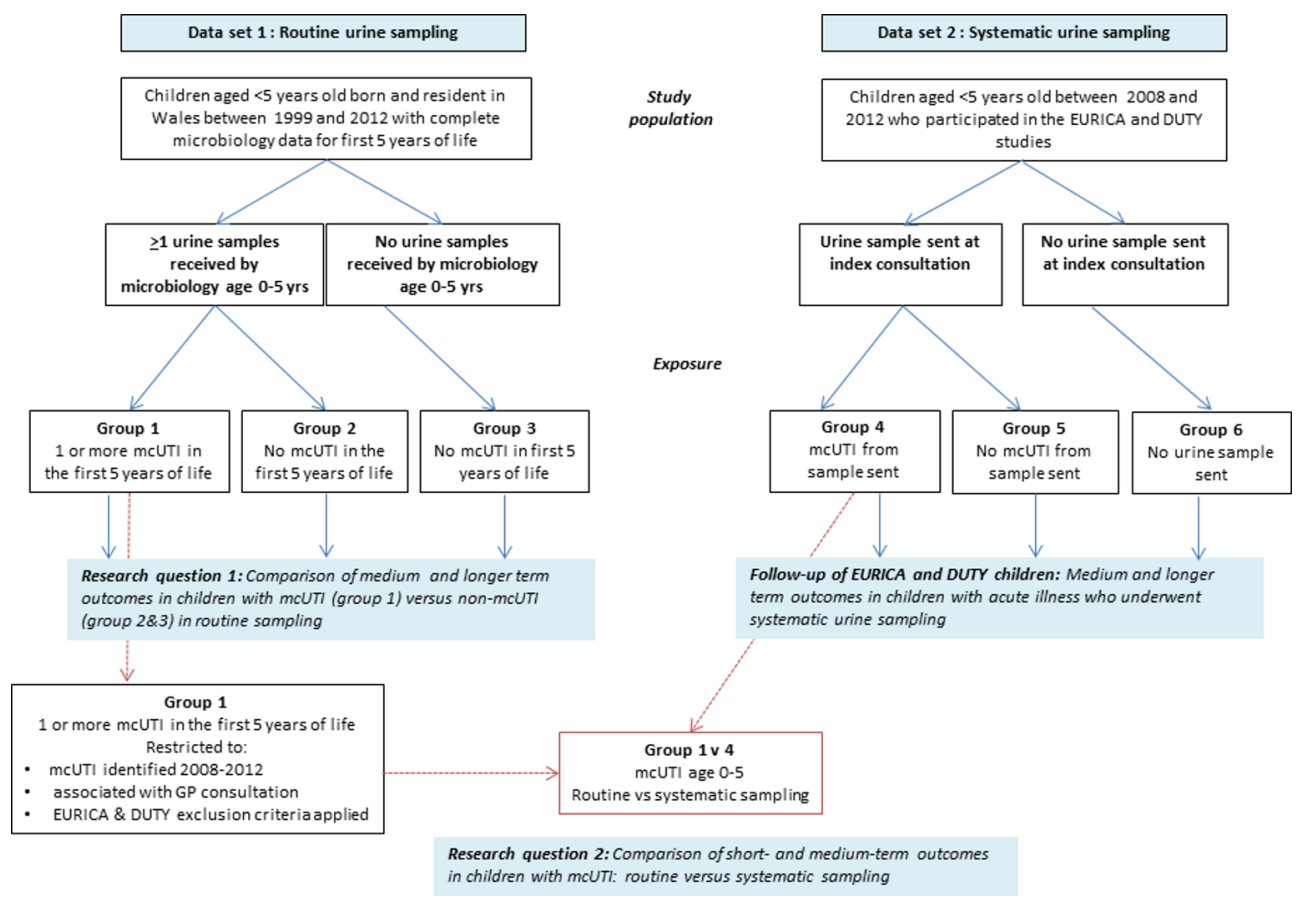

Figure 2 Flow chart of study participants. DUTY, Diagnosis of Urinary Tract infection in Young children; GP, general practice; $\mathrm{mcUTI}$, microbiologically confirmed urinary tract infection. 
when comparing children with an mcUTI from data sets 1 and 2, children in group 1 will be selected to match the DUTY and EURICA study eligibility criteria as closely as possible within the constraints of using routine data. Data sets 1 and 2 will be limited to children with an mcUTI associated with a GP consultation between March 2008 and April 2012. For both data sets, the child's mcUTI, associated with a GP consultation (defined as within 14 days prior to the sample), will be identified and defined as the index consultation. To limit the potential transfer of GP systematic sampling behaviour, children from group 1 with index consultations between 2008 and 2012 at practices which participated in the EURICA study will be flagged as will children with index consultations between 2010 and 2012 at practices which participated in the DUTY study. Children will only be included once in each study period (ie, a child with a sample sent within the EURICA study period could also appear in the DUTY study period). In addition, we will apply the DUTY study exclusion criteria (where possible) to data set 1 to make them directly comparable to data set 2 ; therefore, children will be excluded from the routine sampling cohort if any criterion met:

- Known neurogenic bladder (eg, spina bifida) or previous bladder surgery.

- Prescribed antibiotics in the 7 days prior to presentation.

- Taking immunosuppressant medication.

- Using urinary catheters.

Children with a history of UTI or vesicoureteric reflux (VUR) will not be excluded (and were not excluded from the DUTY or EURICA studies) and we will explore the impact of these risk factors on outcomes.

\section{Exposure}

UTI cases will be based on NHS laboratory results. For data set 1 , this will be through microbiological culture data downloaded from Datastore. These data represent samples (from both community and hospital settings) which have been classified as positive or negative by NHS laboratories according to their standard operating procedures. We do not know how urine was sampled, and this is likely to vary between settings. In most cases, these are likely to be clean catch samples, but may include urine collection pads or bags (particularly in community samples) as recommended by NICE; or catheter or suprapubic aspiration in hospital samples. ${ }^{6}$ NHS laboratories take into consideration the nature of the urine sample in their reporting. ${ }^{23}$ For data set 2, we will use the results of microbiological culture from NHS laboratories collected during the DUTY and EURICA studies as some participants were from England (Datastore is Wales only). For data set 2, the presence of significant bacteriuria (pure or predominant growth of $100000 \mathrm{cfu} / \mathrm{mL}$ of urine) will be used to define UTI.

For data set 1 we define the exposure period as $<5$ years and will be grouped as follows (figure 2):
Group 1: children with at least one mcUTI before their fifth birthday or before outcome of interest.

Group 2: children with at least one urine sample but no mcUTI before their fifth birthday or before outcome of interest.

Group 3: children with no urine samples before their fifth birthday or before outcome of interest.

Exposure is a discrete time-varying covariate (0 (group 2 or 3 no UTI or no sample respectively) until first exposure of group 1 (mcUTI) thereafter) and each patient's exposure status will be taken at the point of each outcome; otherwise the exposure status of the child at their fifth birthday will be taken.

For the main analyses, groups 2 and 3 will be considered together as having no mcUTI. Data set 2 will similarly be divided into three groups based on their index consultation when recruited into the DUTY and EURICA studies:

Group 4: children with an mcUTI.

Group 5: children who had a urine sample but no mcUTI.

Group 6: children who had no urine sample.

\section{Study variables}

Table 2 shows a breakdown of the baseline data and possible covariates available for children and maternal characteristics from the data collection forms for EURICA and DUTY and Welsh Demographic Service, Welsh Electronic Cohort of Children and for a subset with GP records. The study outcomes are summarised in table 3 .

\section{Follow-up}

Follow-up of all children in data sets 1 and 2 will continue until the first occurrence of any of: outcome, migration, death or end of follow-up; and for the subanalysis of GP data, if the patient leaves the GP practice linked to SAIL or the last data collection from the general practice. For the analysis of research question 1, using only children whose whole first 5 years of life were covered by the dates that Datastore was available (excluding children whose first 5 years of life fall outside of Datastore availability) and limiting date of births to 1 January 2005 to 31 December 2009, will mean that the shortest follow-up period will be 7 years. Follow-up will be longer where data are available. For research question 2, we will examine outcomes at 30 days and at 1 year (short term), 1-5 years (medium term) and $>5$ years (long term) after the index consultation.

\section{Analysis}

Sample size

Comparison of renal scarring in children routinely sampled for UTI with and without mcUTI

The sample size is based on the outcome of renal scarring of children with and without mcUTI and taken as $15 \%{ }^{8}$ Using an OR of 1.5 (a conservative effect size of 
Table 2 Child and maternal characteristics

\begin{tabular}{|c|c|c|}
\hline Risk factor & $\begin{array}{l}\text { Data set 1: routine } \\
\text { sampling } \\
\text { Source }\end{array}$ & $\begin{array}{l}\text { Data set 2: systematic sampling } \\
\text { Source }\end{array}$ \\
\hline $\begin{array}{l}\text { Age of child at first urine sample (group } 1 / 2 \text { only) or index } \\
\text { consultation (data set 2) }\end{array}$ & Datastore & EURICA and DUTY study data \\
\hline Gender & WECC & EURICA and DUTY study data \\
\hline Ethnicity & WECC & WECC and DUTY study data \\
\hline Maternal age at birth (years) (category) & WECC & $\begin{array}{l}\text { EURICA and DUTY Welsh participants only: } \\
\text { WECC }\end{array}$ \\
\hline Birth weight (g) (category) & WECC & $\begin{array}{l}\text { EURICA and DUTY Welsh participants only: } \\
\text { WECC }\end{array}$ \\
\hline Ever breast fed & WECC & EURICA and DUTY study data \\
\hline Uropathogen (Enterobacteriaceae or other) (mcUTI only) & Datastore & EURICA and DUTY study data \\
\hline Antimicrobial resistance (mcUTI only) & Datastore & EURICA and DUTY study data \\
\hline
\end{tabular}

\section{Congenital malformations (con mals)}

Known to be associatedwith UTI/renal scarring

PEDW

1. Spina bifida/neurobladder

NA: DUTY exclusion

2. Renal/urinary system con mals including vesicoureteric reflux (VUR)

EURICA and DUTY study data

\section{Possibly associatedwith UTI/renal scarring}

1. Down's syndrome

PEDW; WECC

EURICA and DUTY study data

2. Cerebral palsy/other paralytic syndromes

WECC EURICA and DUTY study data

Congenital malformations-major/minor

WECC

EURICA and DUTY Welsh participants only: WECC

\section{Comorbidities}

1. Diabetes diagnosed under the age of 5 years

PEDW

EURICA and DUTY study data*

2. Renal or urogenital surgery

PEDW EURICA study data* DUTY exclusion

3. Cancer

PEDW

4. Immunosuppressive disease

PEDW

5. Circumcision (aged $<5$ years)

PEDW; GP

EURICA and DUTY study data*

EURICA and DUTY study data*

Factors for follow-up of study participants and research question 2

Symptoms and signs at index consultation

Management at index consultation

Antenatal ultrasound urinary system abnormalities

Family history of UTI/urinary system problems

Recent antibiotics (7 days prior to index consultation)
$-$

GP

$-$

$-$

GP

EURICA and DUTY study data*

*At time of index consultation.

DUTY, Diagnosis of Urinary Tract infection in Young children; EURICA, Welsh cohort study of urinary tract infection in children; GP, general practice; mcUTI, microbiologically confirmed UTI; NA, not applicable; PEDW, Patient Episode Database for Wales; UTI, urinary tract infection; WDS, Welsh Demographic Service; WECC, Welsh Electronic Cohort of Children.

$5 \%$ difference between groups), two-sided alpha 0.05 , power $=90 \%$ and given a ratio of 32:1 (children diagnosed with mcUTI:children with no UTI diagnosis) in the data set, we require 15519 children (452 with
mcUTI:15067 without mcUTI) for analysis. Initial examination of the SAIL data set identified just under 13000 children less than 5 years old with UTI between 1999 and 2012. As our sample size calculation requires 452 with 
Table 3 Study outcomes

\begin{tabular}{lll}
\multicolumn{2}{l}{ Data source } & \\
\hline PEDW & Datastore \\
(All Wales) & GP & (All Wales)
\end{tabular}

Primary outcome
Renal scarring
Sensitivity analyses
Any renal pathology
codes
GP renal scarring codes

Secondary outcomes

\begin{tabular}{lll} 
Hospital admissions & $\checkmark$ & \\
Day cases & $\checkmark$ & \\
Renal/urological surgery & $\checkmark$ & \\
Hypertension & $\checkmark$ & $\checkmark$ \\
Chronic kidney disease & $\checkmark$ & \\
Renal failure & $\checkmark$ & $\checkmark$ \\
UTIs & $\checkmark$ & $\checkmark$ \\
Renal imaging & & $\checkmark$ \\
GP consultations & \\
Antibiotics & \\
$\begin{array}{l}\text { Dysfunctional voiding } \\
\text { Microbiologically confirmed } \\
\text { UTI (5-7 years follow-up) }\end{array}$ & $\checkmark$ \\
\hline
\end{tabular}

GP, general practice; PEDW, Patient Episode Database for Wales; UTI, urinary tract infection.

UTI, we can be confident of adequate power for this study. However, the true proportion with renal scarring is likely to be less than that reported by Shaikh et al. ${ }^{8}$ Assuming a proportion of $8 \%$ with renal scarring in the mcUTI group, and an OR of 1.5 (2.5\% difference), two-sided alpha $=0.05$, power $=90 \%$ and a ratio of $32: 1$, a total of 35833 children (1044 with UTI:34789 without UTI) are required for analysis, which is still achievable.

\section{Comparison of systematically versus routinely sampled UTI}

This sample size is constrained by the number of children with a systematically sampled mcUTI by NHS lab $(n=374)$. Using the lower estimate of renal scarring of $8 \%$ of routinely sampled UTIs, then a $5 \%$ difference $(13 \%$ in the systematically sampled group) would give $89 \%$ power, with a two-sided alpha $=0.05$ and a ratio of 32:1 (routinely sampled UTIs 11 968:systematically sampled UTIs 374).

Both analyses use multivariable regression. Using Green's $^{24}$ formulae, assuming medium effect sizes $(\mathrm{OR}=1.5)$, a two-sided alpha $=0.05$ and power $=80 \%$, to test 20 predictors in the multivariable regression model we require at least 159 children in total. This suggests that we will be adequately powered for both analyses (given these assumptions) to examine predictors of short and medium-term outcomes.

\section{Statistical analysis}

\section{Data set 1: routine sampling of UTI}

Research question 1: Comparison of medium (up to 5 years) and longer term ( $\geq 5$ years) outcomes in children with mcUTI versus those without mcUTI in routine sampling.

Baseline variables will be described using appropriate descriptive summaries (n (\%), mean (SD), median $(\mathrm{IQR})$ ) to summarise the population for the main analyses by groups 1, 2 and 3 and any marked imbalance between the groups will be identified. The main comparative analyses will be carried out at a child level since outcomes relate to an individual's exposure to one or more UTIs and will test the null hypothesis that there are no differences in outcomes in the first 5 years and between the ages of 5 and 7 years. The primary analysis will consist of a comparison of rate of diagnosis of renal scarring (no renal scarring, renal scarring recorded $<5$ years, renal scarring recorded 5-7 years) in children with mcUTI versus children with no mcUTI, using a multinomial regression model. Results will be reported as relative risk ratios alongside $95 \%$ CIs. A survival model will also be performed to model time to first renal scarring diagnosis taking into account competing risks (such as deaths and migration) and differences in time at risk and to allow us to look for this outcome using all available follow-up for each child (at least 7 years). We will estimate HRs with 95\% CIs for each exposure group.

We will construct a directed acyclic graph to inform our choice of variables to include in the analysis. Confounding variables such as those listed in table 2 and also mcUTI that could be considered to be on the causal pathway will be defined a priori. We will run multiple mediation analyses using renal scarring as the dependent variable, mcUTI as the mediation variable and confounders as the independent variables. First we will identify the independent variables associated with renal scarring (using a univariable logistic (where scarring is rare) or log-linear regression model (where scarring is common)) and identify the mediation variables (mcUTI or not) that are associated with the significant independent variables. These will all be included in the mediation model. For each of the significant independent variables, two regression models will be performed with and without the mediation variable. We will calculate the indirect effect (and the effect of the mediator) using the regression coefficients from both regression models.

Unadjusted and adjusted relative risk ratios will be estimated, together with $95 \%$ CIs.

Several sensitivity analyses are proposed: the primary outcome will be expanded to include any renal pathology codes due to uncertainty around whether the renal scarring codes are sufficiently sensitive to pick up all cases of renal scarring. In addition, we will explore using Read codes from GP notes to pick up additional renal scarring diagnoses. Two effect modifiers were identified as a basis for subgroup analyses for the primary outcome: gender of child and presence of any renal/urological congenital 
anomalies. These preplanned analyses will be conducted by the inclusion of appropriate interaction terms in the models.

Secondary outcomes will be analysed using multinomial and time to event models (table 3). Poisson regression models will be used where the outcome is a count of event (eg, hospital admissions, GP consultations, antibiotics prescribed); results will be represented as incidence rate ratios alongside 95\% CIs. Additionally, we will identify risk factors for renal scarring (as above, plus age at first mcUTI, bacterial type and resistance) in children presenting with a childhood mcUTI. In a subsample of children linked to GP data, the 14 days prior to the urine sample submission date will be examined to determine whether there was an associated GP consultation. We will then estimate the likely rate of GP-associated mcUTIs and the rate of mcUTIs submitted from another setting (such as out of hours, outpatients or as a result of a hospital admission). An identical analysis to the primary outcome will be taken to examine whether the risk of renal scarring differs between those with an mcUTI or not, between different settings and the interaction between the two. Where numbers allow, variation in outcome will be accounted for at the level of the general practice in a multilevel model. We will also describe the levels of urine sampling and incidence of mcUTI from general practice in the routine data.

\section{Data set 2: systematic sampling of UTI}

Detailed study data for DUTY and EURICA participants are available, including age, gender and deprivation, presenting features, GP diagnosis and acute management. Recruited children are already grouped into mcUTI or no mcUTI through the study microbiology data and outcomes will be compared according to these groups. Five-year inpatient hospital outcomes will be available for all EURICA and DUTY children (the last participant of DUTY study was recruited in April 2012). We will be able to describe serious short-term (30 days and less than 1 year after index consultation) and medium-term (1-5 years) outcomes, including hospital admission, renal imaging, renal scarring, VUR and renal failure outcomes for all children in this group using Patient Episode Database for Wales data in Wales and NHS Digital hospital data in England. We will be able to describe other outcomes, such as GP consultations, recurrent UTI, dysfunctional voiding, antibiotic prescriptions, hypertension, chronic kidney disease, using GP data on a subset. In Wales, Datastore will also be used to look at the urine culture results and organism resistance profile for subsequent UTIs.

We will describe GP diagnosis from study data versus Read codes and acute management from the routine data in GP records for this cohort for later comparisons and also to explore the validity of using routinely collected data in these cases. We will also assess the validity of using Read codes to diagnose UTI against microbiological culture results and agreement will be measured using the kappa statistic.
Research question 2: Comparison of short and medium-term outcomes in children with mcUTI: routine versus systematic sampling.

We will compare the outcomes in children with mcUTI identified through routine versus systematic sampling. Children's characteristics, presentation factors, acute management and microbiology results will be described for the groups using appropriate summary statistics. We will compare urine sampling and UTI diagnosis in consultations between routine and systematic sampling. In addition, we will describe blood pressure and creatinine levels for each group if recorded and explore whether comparisons can be made.

Previously mentioned short and medium-term outcomes will be described by the two groups of routine versus selective sampling. Predictors of outcome will be examined as before using a multilevel multinomial regression model (no event, 30 days, event 30 days to 1 year, event $1-5$ years) and again where numbers allowed, variation in outcome will be accounted for at the level of the general practice. Associations between covariates previously described and outcome will first be examined. Unadjusted and adjusted relative risk ratios will be estimated, together with $95 \%$ CIs. We will compare blood pressure and creatinine levels (where available) across the groups; we expect these data to be limited so will be exploratory.

A detailed statistical analysis plan will be written prior to database lock. The reporting and presentation of results will be in accordance with the ${ }^{25-27}$ statements to ensure the comprehensive reporting of our observational non-randomised evaluation of a public health intervention. SPSS (Version 25) and Stata (StataCorp, 2017) will be used for all analyses. ${ }^{28}$

\section{Patient and public involvement}

We have a parent representative (Sarah Jones) who has contributed to all stages of this study. She helped organise a parent group to discuss information provided to DUTY and EURICA study participants explaining the study and opt-out mechanism. She also provided input on the study website and on the procedure in place to manage contacts made by the participants. During the drafting of the statistical analysis plan we discussed the planned analyses with her, and she identified which of the analyses that she felt would be of most interest to parents of children with suspected UTI. Results will be disseminated via the study website and other channels with the input from our parent representative.

\section{Ethics and dissemination}

The governance surrounding data set 1 differs from data set 2. Data set 1 is an anonymised data set made available from SAIL Databank with only approval required from the IGRP whereas data set 2 involves the transfer of identifiable data to data providers which requires ethical approval, s251 support, IGRP and IGARD approval. In order to obtain an unbiased sample from the DUTY and EURICA cohorts, an opt-out consent model is being used; 
this was supported by both the ethics panel and the CAG panel as justification for this model of consent.

The LUCI study will report the risk of renal scarring for children with and without childhood mcUTI across the whole of Wales. The linking of routinely collected data sets will give us a large cohort including demographic, hospital inpatient and outpatient, GP and microbiology data, allowing us to define mcUTI cases and describe outcomes for all children from both primary and secondary care. Clarifying the link between UTI, renal scarring and long-term complications will inform the management of acutely ill children in primary care, where the need for urine sampling is unclear. Determining the clinical implications of 'missed' cases of UTI through our comparison of children with mcUTI identified through routine and systematic urine sampling will also help determine the most appropriate urine sampling strategy. This study maximises the benefits of the previously funded DUTY and EURICA cohorts, representing over 8000 acutely ill children recruited from UK primary care. Significant resources were invested by funders, patients and staff to develop these cohorts. Routine data linkage will allow us to determine longer term outcomes for these children and to determine risks of adverse outcomes. It will also pave the way for even longer term follow-up of cohorts of children with UTI (diagnosed both systematically and routinely) which has been identified as a high research priority by NICE.

A lay summary of the results and links to publications will be made available on the University project website. The academic outputs for this study include (1) this protocol paper, (2) main results from research question 1, and (3) main results from research question 2. The findings from this study will be of interest to clinicians and policymakers and may influence the management of acutely ill children and childhood UTI.

\section{Author affiliations}

${ }^{1}$ Centre for Trials Research, Cardiff University, Cardiff, UK

${ }^{2}$ Division of Population Medicine, National Centre for Population Health and Wellbeing Research, Cardiff University, Cardiff, UK

${ }^{3}$ Nuffield Department of Primary Care Health Sciences, University of Oxford, Oxford, UK

${ }^{4}$ Division of Population Medicine, School of Medicine, Wales Centre for Primary and Emergency Care Research (PRIME Centre Wales), Cardiff University, Cardiff, UK

${ }^{5}$ Centre for Academic Primary Care, University of Bristol, Bristol, UK

${ }^{6}$ Microbiology, Public Health Wales, Cardiff, UK

${ }^{7}$ Paediatric Nephrology, Noah's Ark Children's Hospital for Wales, Cardiff, UK

Acknowledgements We acknowledge the support and input from Sarah Jones, our parent representative for the study. We are also grateful to the DUTY and EURICA participants for their agreement for continued use of their data for this study. The Centre for Trials Research receives funding from Health and Care Research Wales and Cancer Research UK. Wales Centre for Primary and Emergency Care Research (PRIME Centre Wales) receives funding from Health and Care Research Wales. The authors are supported by the Farr Institute CIPHER, funded by Arthritis Research UK, the British Heart Foundation, Cancer Research UK, the Economic and Social Research Council, the Engineering and Physical Sciences Research Council, the Medical Research Council, the National Institute of Health Research, the National Institute for Social Care and Health Research (Welsh Assembly Government), the Chief Scientist Office (Scottish Government Health
Directorates), and the Wellcome Trust (MRC grant number MR/K006525/1) and the National Centre for Population Health and Wellbeing Research (NCPHWR).

Contributors $\mathrm{KHu}$ is the chief investigator of the study. All authors have contributed to and are responsible for the final design of the study. FvL-W is responsible for study management. RC-J and ML are responsible for statistical planning and analysis. $\mathrm{LA}$ and $\mathrm{HJ}$ are responsible for the data management. All authors have read and approved the final manuscript (KHu, FvL-W, RC-J, ML, LA, $\mathrm{HJ}, \mathrm{CB}, \mathrm{NAF}, \mathrm{ADH}, \mathrm{MH}, \mathrm{KHO}, \mathrm{SP}$ and JV).

Funding This project has been funded by the Welsh Government through Health and Care Research Wales (project number 1068).

Competing interests None declared.

Patient consent for publication Not required.

Ethics approval Ethics approval of the study has been given by the Research Ethics Committee for Wales (16/WA/0166) and the transfer and use of identifiable data has been approved by the Health Research Authority's (HRA) Confidentiality Advisory Group (CAG) (16/CAG/0114).

Provenance and peer review Not commissioned; externally peer reviewed.

Open access This is an open access article distributed in accordance with the Creative Commons Attribution Non Commercial (CC BY-NC 4.0) license, which permits others to distribute, remix, adapt, build upon this work non-commercially, and license their derivative works on different terms, provided the original work is properly cited, appropriate credit is given, any changes made indicated, and the use is non-commercial. See: http://creativecommons.org/licenses/by-nc/4.0/.

\section{REFERENCES}

1. Craig JC, Williams GJ, Jones M, et al. The accuracy of clinical symptoms and signs for the diagnosis of serious bacterial infection in young febrile children: prospective cohort study of 15781 febrile illnesses. BMJ 2010;340:c1594.

2. Hoberman A, Chao HP, Keller DM, et al. Prevalence of urinary tract infection in febrile infants. J Pediatr 1993;123:17-23.

3. Foxman B. Epidemiology of urinary tract infections: incidence, morbidity, and economic costs. Am J Med 2002;113:5S-13.

4. Hay AD, Birnie K, Busby J, et al. The Diagnosis of Urinary Tract infection in Young children (DUTY): a diagnostic prospective observational study to derive and validate a clinical algorithm for the diagnosis of urinary tract infection in children presenting to primary care with an acute illness. Health Technol Assess 2016;20:1-294.

5. O'Brien K, Edwards A, Hood K, et al. Prevalence of urinary tract infection in acutely unwell children in general practice: a prospective study with systematic urine sampling. Br J Gen Pract 2013;63:e156-64.

6. NICE. Urinary tract infection in under 16s: diagnosis and management: Guidance and guidelines.

7. Butler CC, O'Brien K, Pickles T, et al. Childhood urinary tract infection in primary care: a prospective observational study of prevalence, diagnosis, treatment, and recovery. Br J Gen Pract 2015;65:e217-23.

8. Shaikh N, Ewing AL, Bhatnagar S, et al. Risk of renal scarring in children with a first urinary tract infection: a systematic review. Pediatrics 2010;126:1084-91.

9. Coulthard MG, Lambert HJ, Keir MJ. Occurrence of renal scars in children after their first referral for urinary tract infection. BMJ 1997;315:918-9.

10. Jacobson SH, Eklöf O, Eriksson CG, et al. Development of hypertension and uraemia after pyelonephritis in childhood: 27 year follow up. BMJ 1989;299:703-6.

11. Round J, Fitzgerald AC, Hulme C, et al. Urinary tract infections in children and the risk of ESRF. Acta Paediatr 2012;101:278-82.

12. Smellie JM, Prescod NP, Shaw PJ, et al. Childhood reflux and urinary infection: a follow-up of 10-41 years in 226 adults. Pediatr Nephrol 1998;12:727-36.

13. Craig JC, Williams GJ. Denominators do matter: it's a myth--urinary tract infection does not cause chronic kidney disease. Pediatrics 2011;128:984-5.

14. Salo J, Ikäheimo R, Tapiainen $T$, et al. Childhood urinary tract infections as a cause of chronic kidney disease. Pediatrics 2011;128:840-7.

15. Coulthard MG, Vernon SJ, Lambert HJ, et al. A nurse led education and direct access service for the management of urinary tract infections in children: prospective controlled trial. BMJ 2003;327:656

16. Jadresic L, Cartwright $\mathrm{K}$, Cowie N, et al. Investigation of urinary tract infection in childhood. BMJ 1993;307:761-4. 
17. van der Voort J, Edwards A, Roberts R, et al. The struggle to diagnose UTI in children under two in primary care. Fam Pract 1997;14:44-8.

18. Hollingworth W, Busby J, Butler CC, et al. The Diagnosis of Urinary Tract Infection in Young Children (DUTY) study clinical rule: economic evaluation. Value in Health 2017;20:556-66.

19. Jones $\mathrm{KH}$, Ford DV, Jones $\mathrm{C}$, et al. A case study of the Secure Anonymous Information Linkage (SAIL) gateway: a privacy-protecting remote access system for health-related research and evaluation. $J$ Biomed Inform 2014;50:196-204.

20. Ford DV, Jones KH, Verplancke JP, et al. The SAIL Databank: building a national architecture for e-health research and evaluation. BMC Health Serv Res 2009;9:157.

21. Lyons RA, Jones KH, John G, et al. The SAIL databank: linking multiple health and social care datasets. BMC Med Inform Decis Mak 2009;9:3.

22. Robling M, Bekkers MJ, Bell K, et al. Effectiveness of a nurse-led intensive home-visitation programme for first-time teenage mothers
(Building Blocks): a pragmatic randomised controlled trial. Lancet 2016;387:146-55.

23. Public Health England. UK Standards for Microbiology Investigations. Investigation of Urine. B41. 2018 https://www.gov.uk/government/ publications/smi-b-41-investigation-of-urine.

24. Green SB. How Many Subjects Does It Take To Do A Regression Analysis. Multivariate Behav Res 1991;26:499-510.

25. Gilbert R, Lafferty R, Hagger-Johnson G, et al. GUILD: GUidance for Information about Linking Data sets. J Public Health 2018;40:191-8.

26. von Elm E, Altman DG, Egger M, et al. Strengthening the Reporting of Observational Studies in Epidemiology (STROBE) statement: guidelines for reporting observational studies. $B M J$ 2007:335:806-8.

27. Benchimol El, Smeeth L, Guttmann A, et al. The REporting of studies Conducted using Observational Routinely-collected health Data (RECORD) statement. PLoS Med 2015;12:e1001885.

28. IBM. IBM SPSS Statistics for Windows: Version 22.0.. 2013. 